\title{
QUARK MIXING AND CP VIOLATION — THE CKM MATRIX
}

\author{
ULRICH NIERSTE \\ Institut für Theoretische Teilchenphysik, Universität Karlsruhe, 76128 Karlsruhe, Germany \\ E-mail: nierste@particle.uni-karlsruhe.de
}

\begin{abstract}
I present the status of the elements and parameters of the Cabibbo-Kobayashi-Maskawa (CKM) matrix and summarise the related theoretical progress since Lepton-Photon 2003. One finds $\left|V_{u s}\right|=0.2227 \pm$ 0.0017 from $\mathrm{K}$ and $\tau$ decays and $\left|V_{c b}\right|=(41.6 \pm 0.5) \cdot 10^{-3}$ from inclusive semileptonic $B$ decays. The unitarity triangle can now be determined from tree-level quantities alone and the result agrees well with the global fit including flavour-changing neutral current (FCNC) processes, which are sensitive to new physics. From the global fit one finds the three CKM angles $\theta_{12}=12.9^{\circ} \pm 0.1^{\circ}, \theta_{23}=2.38^{\circ} \pm 0.03^{\circ}$ and $\theta_{13}=0.223^{\circ} \pm 0.007^{\circ}$ in the standard PDG convention. The CP phase equals $\delta_{13} \simeq \gamma=\left(58.8_{-5.8}^{+5.3}\right)^{\circ}$ at $1 \sigma \mathrm{CL}$ and $\gamma=\left(58.8_{-15.4}^{+11.2}\right)^{\circ}$ at $2 \sigma \mathrm{CL}$. A major progress are first results from fully unquenched lattice QCD computations for the hadronic quantities entering the UT fit. I further present the calculation of three-loop QCD corrections to the charm contribution in $K^{+} \rightarrow \pi^{+} \nu \bar{\nu}$ decays, which removes the last relevant theoretical uncertainty from the $K \rightarrow \pi \nu \bar{\nu}$ system. Finally I discuss mixing-induced CP asymmetries in $b \rightarrow s \bar{q} q$ penguin decays, whose naive average is below its Standard Model value by $3 \sigma$.
\end{abstract}

\section{Flavour in the Standard Model}

In the Standard Model transitions between quarks of different generations originate from the Yukawa couplings of the Higgs field to quarks. The non-zero vacuum expectation value $v$ of the Higgs field leads to quark mass matrices $M^{u}$ and $M^{d}$ for the up-type and down-type quarks, respectively. The transformation to the physical mass eigenstate basis, in which the mass matrices are diagonal, involves unitary rotations in flavour space. The rotation of the left-handed down-type quarks relative to the left-handed up-type quarks is the physical Cabibbo-KobayashiMaskawa (CKM) matrix $V$. It appears in the couplings of the $W$ boson to quarks and is the only source of transitions between quarks of different generations. $V$ contains one physical complex phase, which is the only source of $\mathrm{CP}$ violation in flavour-changing transitions.

Flavour physics first aims at the precise determination of CKM elements and quark masses, which are fundamental parameters of the Standard Model. The second target is the search for new physics, pursued by confronting high precision data with the predictions of the Standard Model and its extensions. To this end it is useful to distinguish between charged-current weak decays and flavour-changing neutral current (FCNC) processes. The determination of CKM elements from the tree-level charged-current weak decays, discussed in Sect. 2, is practically unaffected by possible new physics. ${ }^{a}$ By contrast, FCNC processes are very sensitive to virtual effects from new particles with masses at and above the electroweak scale, even beyond $100 \mathrm{TeV}$ in certain models of new physics. FCNC processes are discussed in Sect. 3.

$V$ can be parameterised in terms of three mixing angles $\theta_{12}, \theta_{23}, \theta_{13}$ and one complex phase $\delta_{13}$, which violates CP . Adopting the PDG convention ${ }^{1}$, in which $V_{u d}, V_{u s}, V_{c b}$ and $V_{t b}$ are real and positive, these parameters

\footnotetext{
${ }^{a}$ Still new physics can be revealed if the $3 \times 3 \mathrm{CKM}$ matrix $V$ is found to violate unitarity: One may then infer the existence of new (for example iso-vector) quarks which mix with the known six quarks. Further leptonic decays of charged mesons are tree-level, but sensitive to effects from charged Higgs bosons.
} 


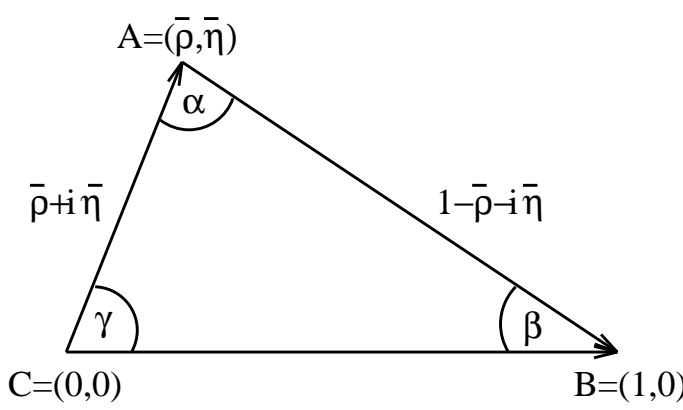

Figure 1. Unitarity triangle (UT).

can be determined through

$V_{u s}=\sin \theta_{12} \cos \theta_{13}, \quad V_{u b}=\sin \theta_{13} e^{-i \delta_{13}}$,

$V_{c b}=\sin \theta_{23} \cos \theta_{13}$.

The Wolfenstein parameterisation ${ }^{2}$

$$
V=\left(\begin{array}{ccc}
1-\frac{\lambda^{2}}{2} & \lambda & A \lambda^{3}(\rho-i \eta) \\
-\lambda & 1-\frac{\lambda^{2}}{2} & A \lambda^{2} \\
A \lambda^{3}(1-\rho-i \eta) & -A \lambda^{2} & 1
\end{array}\right)(2)
$$

is an expansion of $V$ in terms of $\lambda \simeq 0.22$ to order $\lambda^{3}$. It shows both the hierarchy of the CKM elements and their correlations, like $\left|V_{u s}\right| \simeq\left|V_{c d}\right|$ and $\left|V_{c b}\right| \simeq\left|V_{t s}\right|$. The apex of the standard unitarity triangle (UT), which is shown in Fig. 1 is defined by ${ }^{3}$

$$
\bar{\rho}+i \bar{\eta} \equiv-\frac{V_{u b}^{*} V_{u d}}{V_{c b}^{*} V_{c d}}=\left|\frac{V_{u b}^{*} V_{u d}}{V_{c b}^{*} V_{c d}}\right| e^{i \gamma}
$$

$(\bar{\rho}, \bar{\eta})$ coincide with $(\rho, \eta)$ up to corrections of order $\lambda^{2}$. With Eq. (3) and

$$
\lambda \equiv \sin \theta_{12}, \quad A \lambda^{2} \equiv \sin \theta_{23},
$$

the Wolfenstein parameterisation can be made exact ${ }^{3}$, that is $V$ can be expressed in terms of $(\lambda, A, \bar{\rho}, \bar{\eta})$ to any desired order in $\lambda$. In the following I always use the PDG phase convention and the exact definitions in Eqs. (3) and (4), with one exception: I ignore the small phase of $-V_{c d}$ (see Ref. ${ }^{1}$ ), so that I can identify $\arg V_{u b}^{*}=\delta_{13}$ with $\gamma$ and $\arg V_{t d}^{*}$ with the angle $\beta$ of the unitarity triangle. This approximation is correct to $0.1 \%$.

The numerical results presented in the following have been prepared with the help of

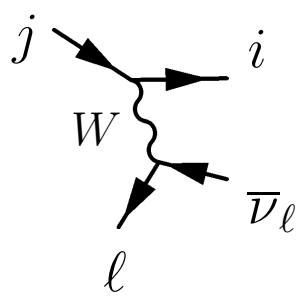

Figure 2. $\left|V_{u j}\right|$ and $\left|V_{c j}\right|, j=d, s, b$, are determined from semileptonic decays.

the Heavy Flavor Averaging Group (HFAG) ${ }^{4}$ and the CKMfitter ${ }^{5}$ and $U_{T F i t}{ }^{6}$ groups. CKMfitter uses a Frequentist treatment of theoretical uncertainties, while UTFit pursues a Bayesian approach, using flat probability distribution functions for theoretical uncertainties.

\section{CKM elements from tree-level decays}

The standard way to determine the magnitudes of the elements of the first two rows of $V$ uses semileptonic hadron decays, depicted in Fig. 2. From Eq. (2) one realizes that an accurate determination of $V_{u s}$ or $V_{u d}$ determines $V_{c d}$ and $V_{c s}$ as well. Therefore measurements of semileptonic $c \rightarrow d$ and $c \rightarrow s$ decays are usually viewed as test of the computation of the hadronic form factors entering the decay amplitudes. Charm decays are covered by Iain Stewart. ${ }^{7}$

\section{$2.1 V_{u d}$}

$V_{u d}$ can be determined from superallowed $\left(0^{+} \rightarrow 0^{+}\right)$nuclear $\beta$ decay and from the $\beta$ decays $n \rightarrow p \ell \bar{\nu}_{\ell}(\gamma)$ and $\pi^{-} \rightarrow \pi^{0} \ell \bar{\nu}_{\ell}(\gamma)$. Since no other decay channels are open, the semileptonic decay rate can be accessed through lifetime measurements. All three methods involve the hadronic form factor of the vector current:

$$
\left\langle f\left|\bar{u} \gamma_{\mu} d\right| i\right\rangle,
$$


where $(i, f)=\left(0^{+}, 0^{+}\right),(n, p)$ or $\left(\pi^{ \pm}, \pi^{0}\right)$. The neutron $\beta$ decay further involves the form factor of the axial vector current:

$$
\left\langle f\left|\bar{u} \gamma_{\mu} \gamma_{5} d\right| i\right\rangle
$$

The form factors parameterise the longdistance QCD effects, which bind the quarks into hadrons. The normalisation of the vector current is fixed at the kinematic point of zero momentum transfer $p_{i}-p_{f}$ in the limit $m_{u}=m_{d}$ of exact isospin symmetry. The Ademollo-Gatto theorem ${ }^{9}$ assures that corrections are of second order in the symmetry breaking parameter $\left(m_{d}-m_{u}\right) / \Lambda_{\text {had }}$, where $\Lambda_{\text {had }}$ is the relevant hadronic scale. No such theorem protects the axial form factor $\left\langle p\left|\bar{u} \gamma_{\mu} \gamma_{5} d\right| n\right\rangle$, but the corresponding parameter $G_{A}$ can be extracted from asymmetries in the Dalitz plot. Experimentally the highest precision in the determination of $V_{u d}$ is achieved in the nuclear $\beta$ decay, but $n \rightarrow p \ell \bar{\nu}_{\ell}(\gamma)$ starts to become competitive. However, there is currently a disturbing discrepancy in the measurement of the neutron lifetime among different experiments. ${ }^{8}$ From a theoretical point of view progress in $n \rightarrow p \ell \bar{\nu}_{\ell}(\gamma)$ and, ultimately, in the pristine $\pi^{-} \rightarrow \pi^{0} \ell \bar{\nu}_{\ell}(\gamma)$ decay are highly desirable to avoid the nuclear effects of $0^{+} \rightarrow 0^{+}$transitions. On the theory side QED radiative corrections must be included to match the experimental accuracy, recently even dominant two-loop corrections to $n \rightarrow p \ell \bar{\nu}_{\ell}(\gamma)$ have been calculated. ${ }^{10}$

The world average for $V_{u d}$ reads $^{11}$ :

$$
V_{u d}=0.9738 \pm 0.0005
$$

\section{2 $V_{u s}$}

$V_{u s}$ can be determined from Kaon and $\tau$ decays. The most established method uses the so-called $K \ell 3$ decays $K^{0} \rightarrow \pi^{-} \ell^{+} \nu_{\ell}, K^{0} \rightarrow$ $\pi^{-} \mu^{+} \nu_{\ell}, K^{+} \rightarrow \pi^{0} \ell^{+} \nu_{\ell}$ and $K^{+} \rightarrow \pi^{0} \mu^{+} \nu_{\ell}$. The decay rates schematically read

$$
\Gamma\left(K \rightarrow \pi \ell^{+} \nu_{\ell}\right) \propto
$$

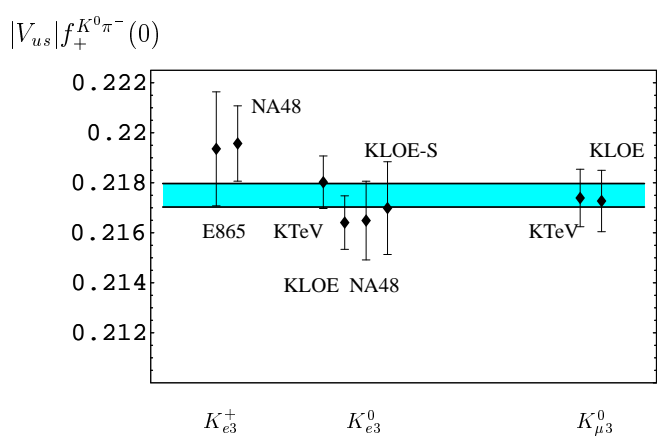

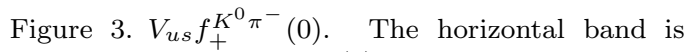
the range quoted in Eq. (6). Courtesy of Vincenzo Cirigliano. ${ }^{11}$

$$
V_{u s}^{2}\left|f_{+}^{K^{0} \pi^{-}}(0)\right|^{2}\left[1+2 \Delta_{S U(2)}^{K}+2 \Delta_{\mathrm{em}}^{K \ell}\right] .
$$

The hadronic physics is contained in

$$
\begin{aligned}
& \left\langle\pi^{-}\left(p_{\pi}\right)\left|\bar{s} \gamma^{\mu} u\right| K^{0}\left(p_{K}\right)\right\rangle= \\
& f_{+}^{K^{0} \pi^{-}}(0)\left(p_{K}^{\mu}+p_{\pi}^{\mu}\right)+\mathcal{O}\left(p_{K}-p_{\pi}\right) \\
& \Delta_{S U(2)}^{K^{+}}=\frac{f_{+}^{K^{+} \pi^{0}}(0)}{f_{+}^{K^{0} \pi^{-}}(0)}-1, \quad \Delta_{S U(2)}^{K^{0}}=0 .
\end{aligned}
$$

and QED corrections are contained in $\Delta_{\mathrm{em}}^{K \ell}$. The Ademollo-Gatto theorem ${ }^{9}$ ensures $f_{+}^{K^{0} \pi^{-}}(0)=1+\mathcal{O}\left(\left(m_{s}-m_{d}\right)^{2} / \Lambda_{\text {had }}^{2}\right)$. $f_{+}^{K^{0} \pi^{-}}(0)-1$ can be calculated with the help of Chiral Perturbation Theory $(\chi \mathrm{PT})^{12}$, which exploits the fact that the pseudoscalar mesons are Goldstone bosons of a dynamically broken chiral symmetry of QCD. $\chi \mathrm{PT}$ amounts to a systematic expansion in $p / \Lambda_{\text {had }}$, $M / \Lambda_{\text {had }}, m_{\ell} / \Lambda_{\text {had }}$ and the electroweak coupling $e$. Here $p$ and $M$ denote meson momenta and masses and $m_{\ell}$ is the lepton mass. There has been a substantial progress in the calculation of both $\Delta_{\mathrm{em}}^{K \ell 13}$ and $f_{+}^{K^{0} \pi^{-}}(0)^{14}$ since LP'03. Significant effects of $\mathcal{O}\left(e^{2} p^{2}\right)$ QED corrections on differential distributions were found; they must be included in Monte Carlo simulations. The value for $V_{u s} f_{+}^{K^{0} \pi^{-}}(0)$ extracted from various experiments is shown in Fig. 3. The world average reads: ${ }^{11}$

$$
f_{+}^{K^{0} \pi^{-}} V_{u s}=0.2175 \pm 0.0008 .
$$


Combining the results from $\chi P T$ at order $p^{6}$ and quenched lattice gauge theory (new) to ${ }^{14}$

$$
f_{+}^{K^{0} \pi^{-}}=0.972 \pm 0.012
$$

one arrives at

$$
V_{u s}=0.2238 \pm 0.0029
$$

from $K \ell 3$.

$V_{u s}$ can also be determined from the $K \mu 2$ decay $K^{+} \rightarrow \mu^{+} \nu_{\mu}(\gamma) .{ }^{15}$ The hadronic quantity entering this decay is the Kaon decay constant $F_{K}$. Uncertainties can be better controlled in the ratio $F_{K} / F_{\pi}$ and one considers

$$
\begin{aligned}
& \frac{\Gamma\left(K^{+} \rightarrow \mu^{+} \nu_{\mu}(\gamma)\right)}{\Gamma\left(\pi^{+} \rightarrow \mu^{+} \nu_{\mu}(\gamma)\right)}= \\
& \frac{V_{u s}^{2}}{V_{u d}^{2}} \frac{F_{K}^{2}}{F_{\pi}^{2}} \frac{M_{K}^{2}-m_{\mu}^{2}}{M_{\pi}^{2}-m_{\mu}^{2}}\left[1-\frac{\alpha}{\pi}\left(C_{\pi}-C_{K}\right)\right]
\end{aligned}
$$

with QED corrections $C_{\pi}-C_{K}=3.0 \pm 1.5$. Using the result ${ }^{16} F_{K} / F_{\pi}=1.210 \pm 0.004 \pm$ 0.013 computed by the MILC collaboration with $2+1$ dynamical quarks, one finds (with $V_{u d}$ from Eq. (5)):

$$
V_{u s}=0.2223 \pm 0.0026
$$

from the $K \mu 2$ decay. This is astonishingly precise and $K^{+} \rightarrow \mu^{+} \nu_{\mu}(\gamma)$ may constrain mass and couplings of a charged Higgs boson, which can mediate this decay as well. ${ }^{15}$

The third possibility to measure $V_{u s}$ used hadronic $\tau$ decays to the inclusive final state with strangeness $|S|=1$. The experimental inputs are the ratios

$$
\begin{aligned}
& R_{\tau s}=\frac{\Gamma^{\Delta S=1}\left(\tau \rightarrow \text { hadrons } \nu_{\tau}(\gamma)\right)}{\Gamma\left(\tau \rightarrow e \bar{\nu}_{e} \nu_{\tau}(\gamma)\right)} \propto V_{u s}^{2} \\
& R_{\tau d}=\frac{\Gamma^{\Delta S=0}\left(\tau \rightarrow \text { hadrons } \nu_{\tau}(\gamma)\right)}{\Gamma\left(\tau \rightarrow e \bar{\nu}_{e} \nu_{\tau}(\gamma)\right)} \propto V_{u d}^{2}
\end{aligned}
$$

Here $S$ is the strangeness. The optical theorem allows to relate $R_{\tau s, d}$ to the QCD current-current correlators $\Pi_{s, d}^{T}$ and $\Pi_{s, d}^{L}$ :

$$
\begin{aligned}
& R_{\tau s, d}=12 \pi \int_{0}^{1} d z(1-z)^{2} \times \\
& \quad\left[(1+2 z) \operatorname{Im} \Pi_{s, d}^{T}(z)+\operatorname{Im} \Pi_{s, d}^{L}(z)\right]
\end{aligned}
$$

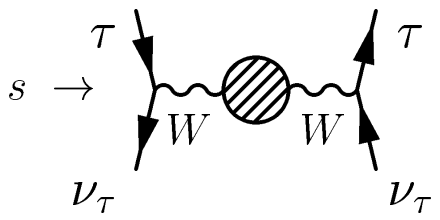

Figure 4. The optical theorem relates $\Gamma^{\Delta S=0,1}(\tau \rightarrow$ hadrons $\left.\nu_{\tau}(\gamma)\right)$ to $\Pi_{s, d}^{T, L}$. The blob denotes the hadronic states contributing to $\Pi_{s, d}^{T, L}$. The leading term in the OPE is obtained by replacing the blob by a $(u, d)$ or $(u, s)$ quark loop and gluons to the desired order in $\alpha_{s}$.

with $z=s / M_{\tau}^{2}=\left(p_{\tau}-p_{\nu_{\tau}}\right)^{2} / M_{\tau}^{2}$. This relationship is depicted in Fig. 4. $\Pi_{s, d}^{T, L}$ can be computed through an operator product expansion (OPE). The leading term is massless perturbative QCD, subleading operators entering $\Pi_{s}^{T, L}$ are $m_{s}^{2}$ and $m_{s}\langle\bar{q} q\rangle$. The OPE amounts to an expansion in $\Lambda_{Q C D} / M_{\tau}$, $m_{s} / M_{\tau}$ and $\alpha_{s}\left(m_{\tau}\right)$. In the limit $m_{s}=0$ of exact $\mathrm{SU}(3)_{\mathrm{F}}$ symmetry the ratio $R_{\tau s} / R_{\tau d}$ would directly determine $V_{u s}^{2} / V_{u d}^{2}$. Hence it suffices to compute the (small) $\mathrm{SU}(3)_{\mathrm{F}}$ breaking quantity ${ }^{17}$

$$
\delta R_{\tau} \equiv \frac{R_{\tau d}}{V_{u d}^{2}}-\frac{R_{\tau s}}{V_{u s}^{2}} .
$$

With $\delta R_{\tau}=0.218 \pm 0.026^{18}$ and experimental data from OPAL ${ }^{19}$ one finds $R_{\tau d}=$ $3.469 \pm 0.014, R_{\tau s}=0.1694 \pm 0.0049$ and finally: ${ }^{18}$

$$
\begin{aligned}
V_{u s} & =\sqrt{\frac{R_{\tau s}}{R_{\tau d} /\left|V_{u d}\right|^{2}-\delta R_{\tau}}} \\
& =0.2219 \pm 0.0033_{\exp } \pm 0.0009_{\mathrm{th}} \\
& =0.2219 \pm 0.0034 .
\end{aligned}
$$

The dominant source of uncertainty in $\delta R_{\tau}$, which enters Eq. (9) as a small correction, is from $m_{s}$. In the near future it should be possible to improve on $V_{u s}$ with data from BaBar and BELLE.

In summary one finds an excellent consistency of the three numbers for $V_{u s}$ from $K \ell 3$, $K \mu 2$ and $\tau$ decays. This is remarkable, since 


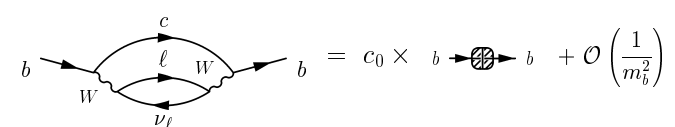

Figure 5. OPE for $\bar{B} \rightarrow X_{c} \ell \bar{\nu}_{\ell}$. The leading operator $\bar{b} b$ has dimension 3 .

the three methods use very different theoretical tools to address the strong interaction: Chiral perturbation theory, lattice gauge theory and the operator product expansion. The result nicely reflects the tremendous progress of our understanding of QCD at low energies. Averaging the results of Eqs. (7), (8) and (9) one finds:

$$
V_{u s}=0.2227 \pm 0.0017
$$

With $V_{u d}$ in Eq. (5) one can perform the firstrow unitarity check

$$
\begin{aligned}
V_{u s}^{2}+V_{u d}^{2}+\left|V_{u b}\right|^{2}-1 & \simeq V_{u s}^{2}+V_{u d}^{2}-1 \\
& =-0.0021 \pm 0.0012
\end{aligned}
$$

The Cabibbo matrix is unitary at the $1.8 \sigma$ level, just as at $L P^{\prime} 03:^{20}$

$$
V_{u s}^{2}+V_{u d}^{2}-1=-0.0031 \pm 0.0017
$$

\section{$2.3 V_{c b}$}

$V_{c b}$ can be determined from inclusive or exclusive $b \rightarrow c \ell \nu_{\ell}$ decays. Exclusive decays are not discussed here. The analysis of the inclusive decay employs an $\mathrm{OPE}^{21}$, similarly to the determination of $V_{u s}$ from $\tau$ decay discussed in Sect. 2.2. The optical theorem relates the inclusive decay rate $\bar{B} \rightarrow X_{c} \ell \bar{\nu}_{\ell}$ to the imaginary part of the $B$ meson self energy, depicted on the LHS of Fig. 5. The OPE matches the self energy diagram to matrix element of effective operators, whose coefficients contain the short-distance information associated with the scale $m_{b}$ and can be calculated perturbatively. Increasing dimensions of the operators on the RHS of Fig. 5 correspond to decreasing powers of $m_{b}$ in the coefficient functions, so that the OPE amounts to a simultaneous expansion in $\Lambda_{Q C D} / m_{b}$ and $\alpha_{s}\left(m_{b}\right)$. Since $\langle B|\bar{b} b| B\rangle=1+\mathcal{O}\left(\Lambda_{Q C D}^{2} / m_{b}^{2}\right)$ and there are no dimension-4 operators, nonperturbative parameters first occur at order $\Lambda_{Q C D}^{2} / m_{b}^{2}$. They are

$$
\begin{aligned}
& \mu_{\pi}^{2} \propto-\left\langle B\left|\bar{b} D_{\perp}^{2} b\right| B\right\rangle \\
& \mu_{G}^{2} \propto\left\langle B\left|\bar{b} i \sigma_{\mu \nu} G^{\mu \nu} b\right| B\right\rangle
\end{aligned}
$$

$\mu_{G}^{2}$, which parameterises the matrix element of the chromomagnetic operator, can be determined from spectroscopy. Hence to order $\Lambda_{Q C D}^{2} / m_{b}^{2}$ one only has to deal with the three quantities $m_{b}, m_{c}$ and $\mu_{\pi}^{2}$, which quantifies the Fermi motion of the $b$ quark inside the $\mathrm{B}$ meson.

The OPE can further be applied to certain spectral moments of the $B \rightarrow X \ell \bar{\nu}_{\ell}$ decay, the distributions of the hadron invariant mass $M_{X}$ and of the lepton energy. Further the same parameters govern different inclusive decays, for instance also $B \rightarrow X_{s} \gamma$. Therefore there is a lot of redundancy in the determination of $V_{c b}$, providing powerful checks of the theoretical framework. The state of the art are fits to order $\Lambda_{Q C D}^{3} / m_{b}^{3}$, which involve 7 parameters. ${ }^{22}$ The result of a global fit to hadron and lepton moments in $B \rightarrow X \ell \nu_{\ell}$ and photon energy moments in $B \rightarrow X_{s} \gamma$ from BaBar, BELLE, CDF, CLEO, DELPHI ${ }^{23}$ can be seen in Fig. 6. It gives

$$
\begin{aligned}
V_{c b}= & 41.6 \pm 0.3_{\exp } \pm 0.3_{\mathrm{OPE} \text { moments }} \\
& \pm 0.3_{\mathrm{OPE} \Gamma_{\mathrm{sl}}} \\
= & (41.6 \pm 0.5) \cdot 10^{-3}
\end{aligned}
$$

from inclusive $B \rightarrow X \ell \nu_{\ell}$.

\section{$2.4\left|V_{u b}\right|$}

I discuss the determination of $\left|V_{u b}\right|$ from inclusive $B \rightarrow X_{u} \ell \nu_{\ell}$ decays. Exclusive decays are discussed in. ${ }^{7}$ In principle one could determine $\left|V_{u b}\right|$ in the same way as $V_{c b}$, if there were no background from $B \rightarrow X_{c} \ell \nu_{\ell}$ decays. Its suppression forces us to impose cuts on the lepton energy $E_{\ell}$, the hadronic energy $E_{X}$, the hadron invariant mass $M_{X}$ 


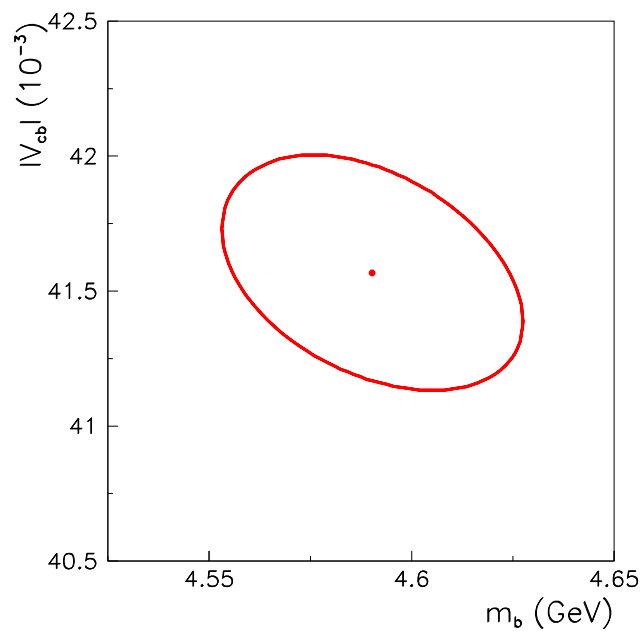

Figure 6. Fit result for $V_{c b}$ vs. $m_{b}$, which is defined in the kinetic scheme. ${ }^{22}$ Fit and plot are courtesy of Oliver Buchmüller and Henning Flächer. See also ${ }^{23}$.

or a judiciously combination of them. $M_{X}$ is too small for an OPE in the portion of phase space passing these cuts. Still some components of the hadron momentum $\vec{P}_{X}$ are large. The description of inclusive $B$ decays in this region involves the non-perturbative shape function $S$, which is a parton distribution function of the B meson. At leading order in $1 / m_{b}$ the same $S$ governs the photon spectrum in $B \rightarrow X_{s} \gamma$ and differential decay rates in $B \rightarrow X_{u} \ell \bar{\nu}_{\ell}$. This allows us to extract $S$ from $B \rightarrow X_{s} \gamma$ for the use in $B \rightarrow X_{u} \ell \bar{\nu}_{\ell}$. The goal to reduce the theoretical uncertainty below $10 \%$ requires to understand corrections in both expansion parameters $\alpha_{s}$ and $\Lambda_{Q C D} / m_{b}$. For a correct treatment of radiative $\mathrm{QCD}$ corrections one must properly relate the differential decay rate $d \Gamma$ to the shape function $S$. This is achieved by a factorisation formula, which has the schematic form: ${ }^{24}$

$$
d \Gamma \propto H \int_{0}^{P_{+}} d \omega J\left(m_{b}\left(P_{+}-\omega\right)\right) S(\omega)
$$

Here $H$ contains the hard QCD, associated with scales of order $m_{b}$. The jet function $J$ and the shape function $S$ contain the physics from scales of orders $M_{X} \sim \sqrt{m_{b} \Lambda_{Q C D}}$ and $\Lambda_{Q C D}$, respectively. $P_{+}$and $P_{-}$are defined as $P_{ \pm}=E_{X} \mp\left|\vec{P}_{X}\right|$. From $\Lambda_{Q C D} \ll P_{+} \sim$ $M_{X} \sim \sqrt{m_{b} \Lambda_{Q C D}} \ll P_{-} \leq m_{b}$ one realizes that one has to deal with a multiscale problem, which is more complicated than $B \rightarrow X_{c} \ell \nu_{\ell}$. The second frontier of research in $B \rightarrow X_{u} \ell \nu_{\ell}$ deals with subleading shape functions $s_{i}$, which occur at order $1 / m_{b}$. They are different in $B \rightarrow X_{u} \ell \nu_{\ell}$ and $B \rightarrow X_{s} \gamma$, but their moments can be related to OPE parameters like $\mu_{\pi}^{2}$, which gives some guidance to model these functions. ${ }^{25}$ Meanwhile an event generator for $B \rightarrow X_{u} \ell \bar{\nu}_{\ell}$ decays is available, ${ }^{26}$ with formulae which contain all available theoretical information and smoothly interpolate between the shape function and OPE regions. It is pointed out that a cut on the variable $P_{+}$, which is directly related to the photon energy in $B \rightarrow X_{s} \gamma$, makes the most efficient use of the $S(\omega)$ extracted from the radiative decay. ${ }^{27,26}$ Alternatively one can eliminate $S(\omega)$ altogether by forming proper weighted ratios of the endpoint photon and lepton spectra in $B \rightarrow X_{s} \gamma$ and $B \rightarrow X_{u} \ell \nu_{\ell}$, respectively. ${ }^{28}$ Using also the information from $B \rightarrow X_{c} \ell \nu_{\ell}$ on $m_{b}$ and $\mu_{\pi}^{2}$ the data from $\mathrm{CLEO}^{29}, \mathrm{BELLE}^{30}$ and $\mathrm{BaBar}^{31}$ combine to the world average ${ }^{4}$

$$
\begin{aligned}
V_{u b} & =\left(4.39 \pm 0.20_{\exp } \pm 0.27_{\mathrm{th}, \mathrm{m}_{\mathrm{b}}, \mu_{\pi}^{2}}\right) \cdot 10^{-3} \\
& =(4.39 \pm 0.34) \cdot 10^{-3}
\end{aligned}
$$

from inclusive $B \rightarrow X_{u} \ell \nu_{\ell}$. Eq. (3) implies that $\left|V_{u b} / V_{c b}\right|$ defines a circle in the $(\bar{\rho}, \bar{\eta})$ plane which is centered around $(0,0)$. With Eqs. (11) and (12) its radius is constrained to

$$
R_{u} \equiv \sqrt{\bar{\rho}^{2}+\bar{\eta}^{2}}=0.45 \pm 0.04 .
$$

\section{$2.5 \arg V_{u b}$}

$\gamma=\arg V_{u b}^{*}$ can be determined from exclusive $B \rightarrow\left(\overrightarrow{D^{0}}\right) X$ decays, where $X$ denotes one or several charmless mesons. This method exploits the interference of the tree-level $b \rightarrow$ $c \bar{u} q$ and $b \rightarrow u \bar{c} q$ amplitudes, where $q=d$ or 

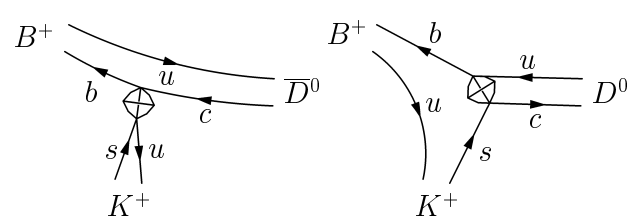

Figure 7. The Gronau-London-Wyler method combines the rates of $B^{ \pm} \rightarrow\left(\bar{D}^{0}\right)\left[\rightarrow f_{i}\right] K^{ \pm}$for different final states $f_{i}$.

$q=s$. The prototype is the Gronau-LondonWyler (GLW) method ${ }^{32}$ shown in Fig. 7. The decays $B \rightarrow D^{0} X$ and $B \rightarrow \overline{D^{0}} X$ interfere, if both subsequent decays $D^{0} \rightarrow f$ and $\overline{D^{0}} \rightarrow f$ are allowed. One needs four measurements to solve for the magnitudes of the $b \rightarrow c$ and $b \rightarrow u$, their relative strong phase and their relative weak phase, which is the desired UT angle $\gamma$. For example one can combine the information of the branching fractions of $B^{+} \rightarrow\left(\overrightarrow{D^{0}}\right)\left[\rightarrow K^{ \pm} \pi^{\mp}\right] K^{+}$and $B^{ \pm} \rightarrow$ $\left(\overrightarrow{D^{0}}\right)\left[\rightarrow \pi^{+} \pi^{-}\right] K^{ \pm}$. This works with untagged non-flavour-specific decays as well: ${ }^{33}$ E.g. the final state $\left(\overrightarrow{D^{0}} \phi\right.$ does not reveal whether the decaying meson was a $B_{s}$ or $\bar{B}_{s}$. Still, when at least three pairs of $\left(\bar{B}_{s}^{)} \rightarrow\left({\overline{D^{0}}}^{2}\left[\rightarrow f_{i}\right] \phi\right.\right.$ and $\left(\bar{B}_{s}\right) \rightarrow\left(\overrightarrow{D^{0}}\left[\rightarrow \bar{f}_{i}\right] \phi\right.$ branching fractions are measured, where $\bar{f}_{i}=C P f_{i}$ (and the $f_{i}$ 's are not $\mathrm{CP}$ eigenstates), one has enough information to solve for $\gamma$. Since no flavour tagging is involved, the Tevatron experiments may contribute to these class of $\gamma$ determinations. The described determination of $\gamma$ from tree-tree interference is modular, that is measurements in different decay modes can be combined, as they partly involve the same hadronic parameters. One should further first average the branching ratios from different experiments and then determine $\gamma$ instead of averaging the inferred values of $\gamma$ obtained from different experiments. Combining (almost) all $B^{+} \rightarrow D^{0} K^{+(*)}$ data gives $(\text { preliminary })^{5}$

$$
\gamma=\left(70_{-14}^{+12}\right)^{\circ}
$$

and the second solution $\gamma-180^{\circ} \sim-110^{\circ}$. This is $\gamma=\arg V_{u b}^{*}$ determined from the treelevel $b \rightarrow u \bar{c} s$ amplitude.

Within the Standard Model $b \rightarrow u \bar{u} d$ decays of tagged $B^{0}$ mesons are used to determine the UT angle $\alpha . b \rightarrow u \bar{u} d$ decays involve both a tree and a penguin amplitude. The penguin component can be eliminated, if several decay modes related by isospin are combined, as in the Gronau-London method ${ }^{34}$ which uses $B^{+} \rightarrow \pi^{+} \pi^{0}, B^{0} \rightarrow \pi^{+} \pi^{-}$and $B^{0} \rightarrow \pi^{0} \pi^{0}$. The $B \rightarrow \rho \pi$ and $B \rightarrow \rho \rho$ decay modes are better suited for the determination of $\alpha$, because the penguin amplitude is smaller. A combined analysis of the $\pi \pi, \rho \pi$ and $\rho \rho$ systems gives

$$
\alpha_{\exp }=\left(99_{-9}^{+12}\right)^{\circ}
$$

and the second solution $\alpha_{\exp }-180^{\circ} \sim-81^{\circ}$. The experimental result $\alpha_{\exp }$ could differ from the true $\alpha=\arg \left(-V_{t b}^{*} V_{t d} /\left(V_{u b}^{*} V_{u d}\right)\right)$, if new physics alters the $\mathrm{B}_{\mathrm{d}}-\overline{\mathrm{B}}_{\mathrm{d}}$ mixing amplitude. However, the influence from new physics is fully correlated in $\alpha_{\exp }$ and the $\mathrm{CP}$ asymmetry measured in $b \rightarrow c \bar{c} s$ decays. From the latter (see Eq. (21) below) we infer the $\mathrm{B}_{\mathrm{d}}-\overline{\mathrm{B}}_{\mathrm{d}}$ mixing phase $2 \beta_{\exp }=(43.7 \pm$ 2.4 $)^{\circ}$. The $\mathrm{B}_{\mathrm{d}}-\overline{\mathrm{B}}_{\mathrm{d}}$ mixing phase cancels from the combination $2 \gamma=360^{\circ}-2 \alpha_{\exp }-2 \beta_{\exp }$, so that one obtains

$$
\gamma=\left(59_{-12}^{+9}\right)^{\circ}
$$

and the second solution $\gamma-180^{\circ} \sim-121^{\circ}$. Since the isospin analysis eliminates the penguin component, this is $\gamma=\arg V_{u b}^{*}$ determined from the tree-level $b \rightarrow u \bar{u} d$ amplitude.

The results in Eqs. (14) and (16) are in good agreement. Their naive average is

$$
\gamma=\left(63_{-9}^{+7}\right)^{\circ} .
$$

The successful determination of a $\mathrm{CP}$ phase from a tree-level amplitude is a true novel result compared to $L P^{\prime} 03$. For the first time we 


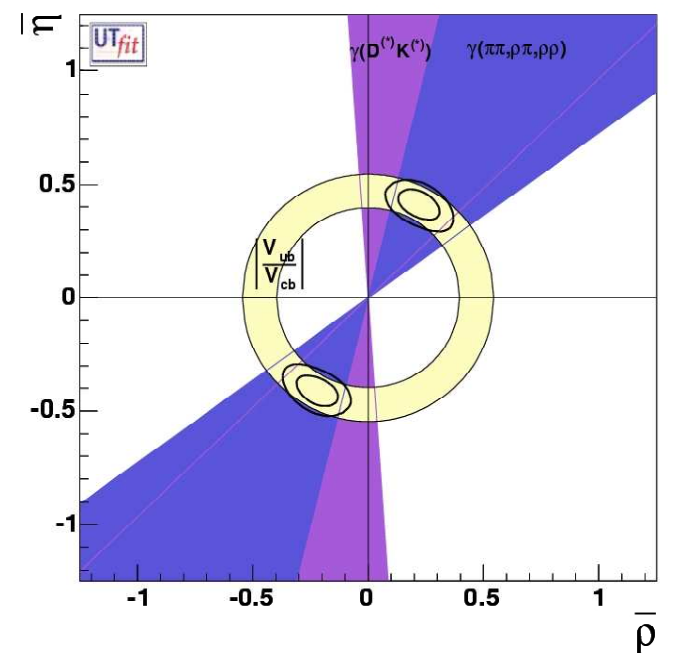

Figure 8. UT from tree quantities alone. The annulus is the constraint in Eq. (13) derived from $\left|V_{u b}\right|$. The dark shadings correspond to $\gamma$ from Eqs. (14) and (16). Courtesy of Maurizio Pierini.

can determine the UT from tree-level quantities alone, the result is shown in Fig. 8. This is important, because the tree-level UT can only be mildly affected by new physics and therefore likely determines the true values of $\bar{\rho}$ and $\bar{\eta}$.

\section{CKM elements from FCNC processes}

In the Standard Model FCNC processes are suppressed by several effects: First they only proceed through electroweak loops. Second they come with small CKM factors like $\left|V_{t s}\right| \sim 0.04$ and $\left|V_{t d}\right| \sim 0.01$. Loops with an internal charm quark are further suppressed by a factor of $m_{q}^{2} / M_{W}^{2}$ from the GIM mechanism. Radiative and leptonic decays further suffer from an additional helicity suppression, because only left-handed quarks couple to $\mathrm{W}$ bosons and undergo FCNC transitions. All these suppression mechanism are accidental, resulting from the particle content of the Standard Model and the unexplained smallness of most Yukawa couplings. They

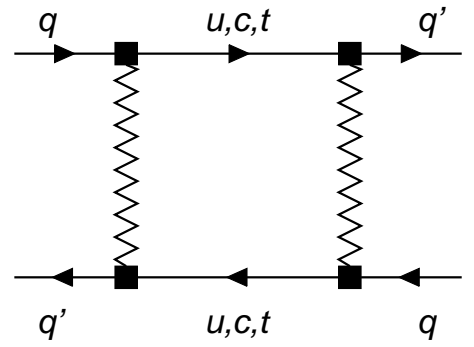

Figure 9. Meson-antimeson mixing. $\left(q, q^{\prime}\right)=(s, d)$, $(b, d)$ and $(b, s)$ for $\mathrm{K}-\overline{\mathrm{K}}$ mixing, $\mathrm{B}_{\mathrm{d}}-\overline{\mathrm{B}}_{\mathrm{d}}$ mixing and $\mathrm{B}_{\mathrm{s}}-\overline{\mathrm{B}}_{\mathrm{S}}$ mixing, respectively.

are absent in generic extensions of the Standard Model (like its supersymmetric generalisations) making FCNC highly sensitive to new physics, probing scales in the range of $200 \mathrm{GeV}$ to $100 \mathrm{TeV}$, depending on the model considered. This feature is a major motivation for the currently performed high-statistics experiments in flavour physics. Comparing different constraints on the UT from FCNCs processes and the tree-level constraints discussed in Sect. 2 therefore provides a very powerful test of the Standard Model.

\subsection{Meson-antimeson mixing}

$\mathrm{K}-\overline{\mathrm{K}}$ mixing, $\mathrm{B}_{\mathrm{d}}-\overline{\mathrm{B}}_{\mathrm{d}}$ mixing and $\mathrm{B}_{\mathrm{s}}-\overline{\mathrm{B}}_{\mathrm{s}}$ mixing are all induced by box diagrams, depicted in Fig. 9. Each meson-antimeson system involves two mass eigenstates, their mass difference $\Delta m$ measures the magnitude of the box diagram and therefore constrains magnitudes of CKM elements. The phase of box diagram and thereby the phases of the CKM elements involved are constrained through CPviolating quantities. Tab. 1 shows the relationship of the measurements to the CKM phenomenology. The quantities in the first two columns of Tab. 1 are well-measured and there is a lower bound on $\Delta m_{B_{s}}$. 


\begin{tabular}{l|ccc} 
& $\mathrm{K}-\overline{\mathrm{K}}$ mixing & $\mathrm{B}_{\mathrm{d}}-\overline{\mathrm{B}}_{\mathrm{d}}$ mixing & $\mathrm{B}_{\mathrm{s}}-\overline{\mathrm{B}}_{\mathrm{s}}$ mixing \\
\hline CP-conserving quantity: & $\Delta m_{K}$ & $\Delta m_{B_{d}}$ & $\Delta m_{B_{s}}$ \\
CKM information: & $\left|V_{c s} V_{c d}\right|^{2}$ & $\left|V_{t b} V_{t d}\right|^{2}$ & $\left|V_{t b} V_{t s}\right|^{2}$ \\
UT constraint: & none & $R_{t}=\sqrt{(1-\bar{\rho})^{2}+\bar{\eta}^{2}}$ & none \\
\hline CP-violating quantity: & $\epsilon_{K}$ & $a_{\mathrm{CP}}^{\operatorname{mix}}\left(B_{d} \rightarrow J / \psi K_{S}\right)$ & $a_{\mathrm{CP}}^{\operatorname{mix}}\left(B_{s} \rightarrow J / \psi \phi\right)$ \\
CKM information: & $\operatorname{Im}\left(V_{t s} V_{t d}^{*}\right)^{2}$ & $\sin (2 \beta)$ & $\sin \left(2 \beta_{s}\right)$ \\
UT constraint: & $\bar{\eta}[(1-\bar{\rho})+$ const. $]$ & $\overline{\bar{\eta}}$ & $\bar{\eta}$
\end{tabular}

Table 1. Relationship of meson-antimeson mixing to CKM and UT parameters. $\beta=\arg V_{t d}^{*}$ is one of the UT angles in Fig. 1 and $\beta_{s}=\arg \left(-V_{t s}\right) \simeq \lambda^{2} \bar{\eta}$.

\section{2 $\epsilon_{K}$}

While $\epsilon_{K}$, which quantifies indirect $\mathrm{CP}$ violation in $K \rightarrow \pi \pi$ decays, is measured at the percent level, its relationship to $\operatorname{Im} V_{t d}^{* 2} \propto$ $\bar{\eta}(1-\bar{\rho})$ is clouded by hadronic uncertainties in the matrix element

$$
\left\langle K^{0}\left|\bar{d} s_{V-A} \bar{d} s_{V-A}\right| \bar{K}^{0}\right\rangle \equiv \frac{8}{3} f_{K}^{2} M_{K}^{2} B_{K} .
$$

This defines the hadronic parameter $B_{K}$, which must be computed by non-perturbative methods like lattice QCD. $M_{K}$ and $f_{K}$ are the well-known mass and decay constant of the Kaon. This field has experienced a major breakthrough since $L P^{\prime} 03$, since meanwhile fully unquenched computations with $2+1 \mathrm{dy}$ namical staggered quarks are available. Using MILC configurations the HPQCD collaboration reports a new result ${ }^{35 b}$

$$
\begin{aligned}
& B_{K}(\mu=2 \mathrm{GeV})= \\
& 0.618 \pm 0.018_{\text {stat }} \pm 0.019_{\text {chiral extrapolation }} \\
& \quad \pm 0.030_{\text {discret. }} \pm 0.130_{\text {pert. matching }} \\
& =0.618 \pm 0.136
\end{aligned}
$$

in the $\overline{\mathrm{MS}}-\mathrm{NDR}$ scheme. The conventionally used renormalisation scale and scheme independent parameter reads

$$
\widehat{B}_{K}=0.83 \pm 0.18
$$

The uncertainty from the perturbative lattice-continuum matching dominates over

${ }^{b}$ In my talk I reported the preliminary value $B_{K}=0.630 \pm 0.018_{\text {stat }} \pm 0.015_{\text {ch. extr. }} \pm 0.030_{\text {disc. }} \pm$ 0.130 p. match. the statistical error and the errors from chiral extrapolation and discretisation in Eq. (18). This matching calculation was performed $i^{36}$. The error in Eq. (18) is a conservative estimate of the unknown two-loop contributions to this matching. If one instead takes twice the square of the one-loop result of ${ }^{36}$ as an estimate of the uncertainty, one finds 0.036 instead of 0.130 in Eq. (18) and

$$
\begin{aligned}
B_{K}(\mu=2 \mathrm{GeV}) & =0.618 \pm 0.054 \\
\widehat{B}_{K} & =0.83 \pm 0.07
\end{aligned}
$$

$\epsilon_{K}$ fixes $\bar{\eta}(1-\bar{\rho})$, so that it defines a hyperbola in the $(\bar{\rho}, \bar{\eta})$ plane.

\section{3 $V_{t d}$ from $\mathrm{B}_{\mathrm{d}}-\overline{\mathrm{B}}_{\mathrm{d}}$ mixing}

The $\mathrm{B}_{\mathrm{d}}-\overline{\mathrm{B}}_{\mathrm{d}}$ mixing mixing amplitude involves the hadronic matrix element

$$
\left\langle B^{0}\left|\bar{b} d_{V-A} \bar{b} d_{V-A}\right| \bar{B}^{0}\right\rangle=\frac{8}{3} M_{B_{d}}^{2} f_{B_{d}}^{2} B_{B_{d}} .
$$

Since the decay constant $f_{B_{d}}$ is not measured, the whole combination $f_{B_{d}}^{2} B_{B_{d}}$ must be obtained from lattice QCD. The hadronic matrix element, however, cancels from the "gold-plated" mixing induced CP asymmetry $a_{\mathrm{CP}}^{\operatorname{mix}}\left(B_{d} \rightarrow J / \psi K_{S}\right)$, which determines $\beta=\arg V_{t d}^{*}$ essentially without hadronic uncertainties. Combining all data from $b \rightarrow c \bar{c} s$ modes results in 37,4

$$
\begin{aligned}
& \sin (2 \beta)=0.69 \pm 0.03, \quad \cos (2 \beta)>0 \\
\Rightarrow \quad & \arg \left( \pm V_{t d}^{*}\right)=\beta=(21.8 \pm 1.2)^{\circ} . \quad(21)
\end{aligned}
$$

The precisely measured $\Delta m_{B_{d}}=0.509 \pm$ $0.004 \mathrm{ps}^{-1}$ is proportional to $\left|V_{t d}\right|^{2} f_{B_{d}}^{2} B_{B_{d}}$. 
The HPQCD collaboration has computed $f_{B_{d}}=216 \pm 22 \mathrm{MeV}$ with $2+1$ dynamical staggered quarks. ${ }^{38}$ This measurement is discussed in detail in ${ }^{7}$. Combining this with $B_{B_{d}}$ from older quenched calculations results in $f_{B_{d}} \sqrt{\widehat{B}_{B_{d}}}=(246 \pm 27) \mathrm{MeV}$, where $\widehat{B}_{B_{d}}=$ $1.52 B_{B_{d}}\left(\mu=m_{b}\right)$ is the conventionally used scale and scheme independent variant of $B_{B_{d}}$. Then from $\Delta m_{B_{d}}$ alone we find

$$
\left|V_{t d}\right|=0.0072 \pm 0.0008
$$

where the error is reduced by a factor of $2 / 3$ compared to the old determination from quenched lattice QCD.

\section{$3.4\left|V_{t d}\right| /\left|V_{t s}\right|$ from $\mathrm{B}-\overline{\mathrm{B}}$ mixing}

A measurement of the ratio $\Delta m_{B_{d}} / \Delta m_{B_{s}}$ will determine $\left|V_{t d}\right| /\left|V_{t s}\right|$ via

$$
\left|\frac{V_{t d}}{V_{t s}}\right|=\sqrt{\frac{\Delta m_{B_{d}}}{\Delta m_{B_{s}}}} \sqrt{\frac{M_{B_{s}}}{M_{B_{d}}}} \xi
$$

with the hadronic quantity $\xi=f_{B_{s}} \sqrt{\widehat{B}_{B_{s}}} /\left(f_{B_{d}} \sqrt{\widehat{B}_{B_{d}}}\right)$ which equals $\xi=1$ in the limit of exact $\mathrm{SU}(3)_{\mathrm{F}}$. A new unquenched HPQCD result for $f_{B_{s}} / f_{B_{d}}{ }^{38}$ presented in ${ }^{7}$ can be used to refine the prediction for $\xi$. The lower bound $\Delta m_{B_{s}} \geq 14.5 \mathrm{ps}^{-1}$ implies $\left|V_{t d} / V_{t s}\right| \leq 0.235$ which constrains one side of the unitarity triangle:

$$
R_{t} \equiv \sqrt{(1-\bar{\rho})^{2}+\bar{\eta}^{2}}=\left|\frac{V_{t d}}{V_{t s} \lambda}\right| \leq 1.06
$$

\subsection{Global fit to the unitarity triangle}

The result of a global fit of $(\bar{\rho}, \bar{\eta})$ to state-ofthe-art summer-2005 data is shown in Fig. 10. It uses $\widehat{B}_{K}=0.85 \pm 0.02 \pm 0.07$ where the first error is Gaussian and the second is scanned over according to the standard CKMfitter $\operatorname{method}^{5}$. For the remaining input $\mathrm{see}^{5}$. The fit output is summarised in this table:

\begin{tabular}{lcc}
\hline quantity & central $\pm \mathrm{CL} \equiv 1 \sigma$ & $\pm \mathrm{CL} \equiv 2 \sigma$ \\
\hline $\bar{\rho}$ & $0.204_{-0.033}^{+0.035}$ & ${ }_{-0.069}^{+0.095}$ \\
$\bar{\eta}$ & $0.336_{-0.021}^{+0.021}$ & ${ }_{-0.060}^{+0.045}$ \\
\hline$\alpha(\mathrm{deg})$ & $98.4_{-5.6}^{+6.1}$ & ${ }_{-11.8}^{+16.8}$ \\
$\beta(\mathrm{deg})$ & $22.77_{-0.83}^{+0.87}$ & ${ }_{-2.04}^{+1.92}$ \\
$\gamma(\mathrm{deg})$ & $58.8_{-5.8}^{+5.3}$ & ${ }_{-15.4}^{+11.2}$ \\
$\left|V_{u b}\right|\left[10^{-3}\right]$ & $3.90_{-0.12}^{+0.12}$ & ${ }_{-0.24}^{+0.29}$ \\
$\left|V_{t d}\right|\left[10^{-3}\right]$ & $8.38_{-0.44}^{+0.32}$ & ${ }_{-1.29}^{+0.56}$
\end{tabular}

The output of the global fit agrees well with the pure tree-level determinations in Eqs. (12) and (17) and Fig. 8.

We can use Eq. (1) to determine $\theta_{13}=$ $0.223^{\circ} \pm 0.007^{\circ}$ from the fitted $\left|V_{u b}\right|$ in the table. With Eq. (1) one finds $\theta_{12}=12.9^{\circ} \pm$ $0.1^{\circ}$ from Eq. (10) and $\theta_{23}=2.38^{\circ} \pm 0.03^{\circ}$ from Eq. (11). Since $1-\cos \theta_{13}$ is negligibly small, the Wolfenstein parameters $\lambda$ and $A \lambda^{2}$ defined in Eq. (4) are simply given by $V_{u s}$ in Eq. (10) and $V_{c b}$ in Eq. (11), respectively.

\section{$3.6 \quad K \rightarrow \pi \nu \bar{\nu}$}

The rare decays $K^{+} \rightarrow \pi^{+} \nu \bar{\nu}$ and $K_{L} \rightarrow$ $\pi^{0} \nu \bar{\nu}$ provide an excellent opportunity to determine the unitarity triangle from $s \rightarrow d$ transitions. With planned dedicated experiments $(\bar{\rho}, \bar{\eta})$ can be determined with a similar precision as today from $b \rightarrow d$ and $b \rightarrow u$ transitions at the $\mathrm{B}$ factories. This is a unique and very powerful probe of the CKM picture of FCNCs. $\operatorname{Br}\left(K_{L} \rightarrow \pi^{0} \nu \bar{\nu}\right)$ is proportional to $\bar{\eta}^{2}$ and dominated by the top contribution. The theoretical uncertainty of the next-to-leading order (NLO) prediction $^{40}$ is below $2 \% . \operatorname{Br}\left(K^{+} \rightarrow \pi^{+} \nu \bar{\nu}\right)$ defines an ellipse in the $(\bar{\rho}, \bar{\eta})$ plane and has a sizeable charm contribution, which inflicts a larger theoretical uncertainty on the next-toleading order (NLO) prediction $^{41}$, leading to $\mathcal{O}(5-10 \%)$ uncertainties in extracted CKM parameters. Parametric uncertainties from $V_{c b}$ and $m_{t}$ largely drop out, if $\sin (2 \beta)$ is calculated from $\operatorname{Br}\left(K_{L} \rightarrow \pi^{0} \nu \bar{\nu}\right)$ and $\operatorname{Br}\left(K^{+} \rightarrow\right.$ 

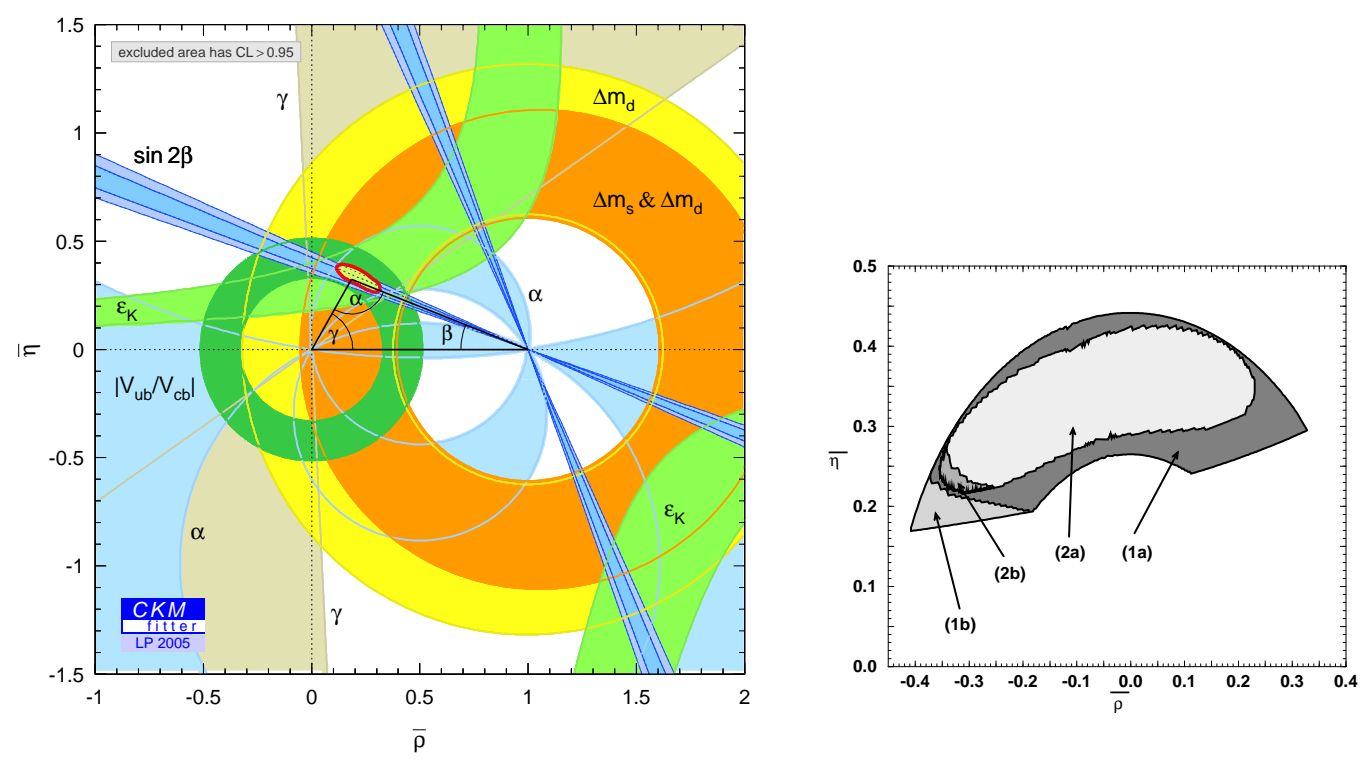

Figure 10. LHS: The UT from a global fit to summer 2005 data. RHS: The first UT fit using theoretical expressions with NLO QCD corrections, performed in $1995 .{ }^{39}$ At that time only $\left|V_{u b}\right|, \epsilon_{K}$ and $\Delta m_{B_{d}}$ could be used. Region 1a corresponds to a scan over $1 \sigma$ ranges of the input parameters.

$\left.\pi^{+} \nu \bar{\nu}\right)$. Therefore the comparison of $\sin (2 \beta)$ determined in Eq. (21) from the B system with $\sin (2 \beta)$ inferred from $K \rightarrow \pi \nu \bar{\nu}$ constitutes a pristine test of the Standard Model. ${ }^{42}$ The impact of a future $10 \%$ measurements of these rare decay modes on the UT is shown in Fig. 11.

The charm contribution is expanded in two parameters: $m_{K}^{2} / m_{c}^{2}$ and $\alpha_{s}\left(m_{c}\right)$. The calculations of $\mathcal{O}\left(m_{K}^{2} / m_{c}^{2}\right)$ corrections was recently completed, finding a $7 \%$ increase of $\mathrm{Br}\left(K^{+} \rightarrow \pi^{+} \nu \bar{\nu}\right)$ with a small residual uncertainty. ${ }^{43} \mathrm{~A}$ new result are the next-tonext-to-leading order (NNLO) QCD corrections to the charm contribution. ${ }^{44}$ This threeloop calculation reduces the theoretical error from unknown higher-order terms well below the parametric uncertainty from $m_{c}$. The branching ratio is now predicted as

$$
\operatorname{Br}\left(K^{+} \rightarrow \pi^{+} \bar{\nu} \nu\right)=(8.0 \pm 1.1) \cdot 10^{-11} .
$$

At NNLO one finds the following reduced theoretical uncertainties for parameters extracted from $\operatorname{Br}\left(K_{L} \rightarrow \pi^{0} \nu \bar{\nu}\right)$ and $\operatorname{Br}\left(K^{+} \rightarrow\right.$

$$
\begin{aligned}
& \left.\pi^{+} \nu \bar{\nu}\right):{ }^{44} \\
& \frac{\delta\left|V_{t d}\right|}{\left|V_{t d}\right|}=0.010, \quad \delta \sin (2 \beta)=0.006, \quad \delta \gamma=1.2^{\circ}
\end{aligned}
$$

\section{CP violation in $b \rightarrow s$ penguin decays}

Within the Standard Model the mixinginduced CP asymmetries in $b \rightarrow s \bar{q} q$ penguin amplitudes are proportional to $\sin (2 \beta)^{\mathrm{eff}}$ which equals $\sin (2 \beta)$ in Eq. (21) up to small corrections from a penguin loop with an up quark. In $b \rightarrow s \bar{u} u$ decays there is also a color-suppressed tree amplitude. In any case the corrections are parametrically suppressed by $\left|V_{u b} V_{u s} /\left(V_{c b} V_{c s}\right)\right| \sim 0.025$. The experimental situation is shown in Fig. 12. A naive average of the measurements of Fig. 12 gives

$$
\sin (2 \beta)^{\mathrm{eff}}=0.51 \pm 0.06
$$

which is below the value of $\sin (2 \beta)$ from treelevel $b \rightarrow \bar{c} c s$ decays in Eq. (21) by $3 \sigma$. Moreover QCD factorisation finds a small and positive correction to $\sin \left(2 \beta_{\text {eff }}\right)-\sin (2 \beta)$ from up- 


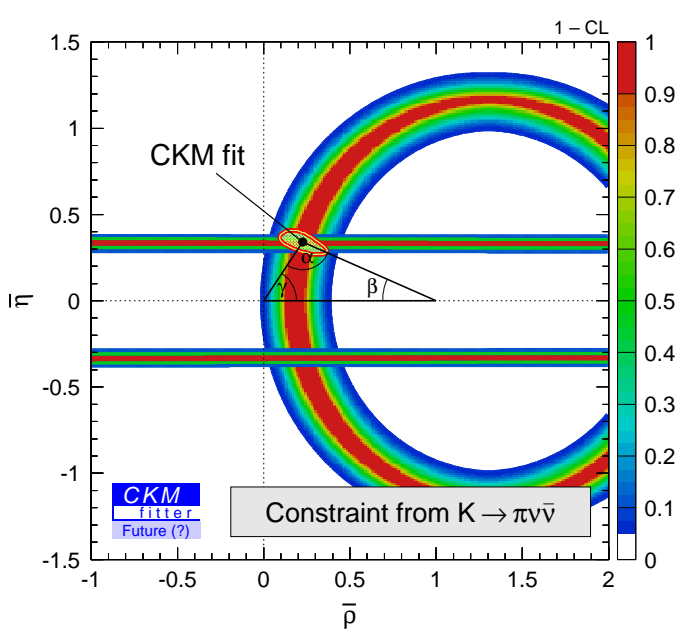

Figure 11. Unitarity triangle from a future measurement of $\operatorname{Br}\left(K_{L} \rightarrow \pi^{0} \nu \bar{\nu}\right)$ and $\operatorname{Br}\left(K^{+} \rightarrow \pi^{+} \nu \bar{\nu}\right)$ with $10 \%$ accuracy. The current UT from the 2005 global fit ${ }^{5}$ is overlaid. The comparison of this UT constructed from $s \rightarrow d$ FCNCs with the UT found from $\mathrm{b}$ decays probes the CKM origin of FCNCs precisely and in a unique way.

\section{$\sin \left(2 \beta^{\text {eff }}\right) / \sin \left(2 \phi_{1}^{\text {eff }}\right) \underset{\text { HFAG }}{\text { HF } 2005}$}

PRELIMINARY

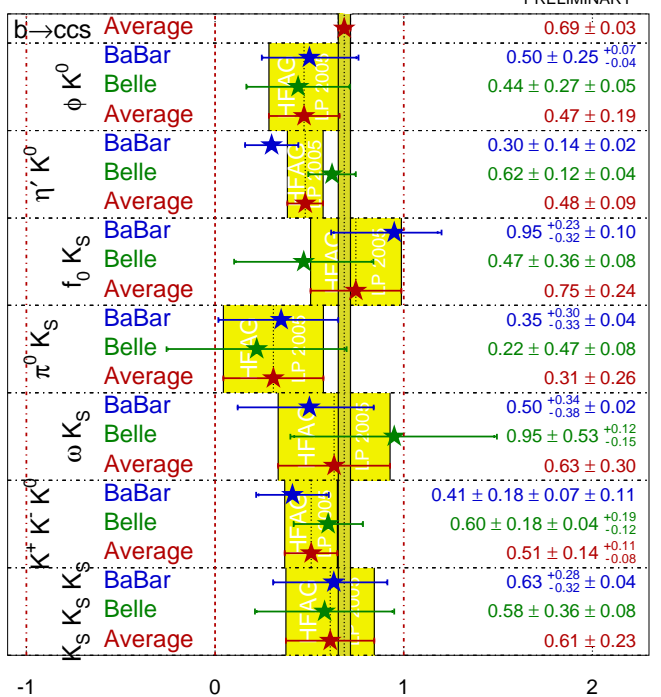

Figure 12. $\sin (2 \beta)^{\text {eff }}$ from various penguin decays. The small vertical yellow band is $\sin (2 \beta)$ from Eq. $(21){ }^{4}$ quark effects. ${ }^{45}$ While the significance of the deviation has decreased since the winter 2005 conferences, the mixing-induced CP asymmetries in $b \rightarrow s \bar{q} q$ decays stay interesting as they permit large effects from new physics. While in $B_{d}$ decays the needed interference of a $B_{d}$ and $\bar{B}_{d}$ decay to the same final state requires a neutral $\mathrm{K}$ meson in the final state, $b \rightarrow s \bar{q} q$ decays of $B_{s}$ mesons go to a flavourless $\bar{s} s \bar{q} q$ state, so that the desired CP effects can be studied in any final state. Hence $B_{s}$ physics has the potential to become the "El Dorado" of $b \rightarrow s \bar{q} q$ penguin physics.

\section{Acknowledgments}

This presentation was made possible through the input and help from Martin Beneke, Ed Blucher, Tom Browder, Oliver Buchmüller, Christine Davies, Henning Ulrik Flächer, Paolo Franzini, Tim Gershon, Martin Gorbahn, Ulrich Haisch, Christopher Hearty, Matthias Jamin, Bob Kowalewski, Heiko Lacker, Zoltan Ligeti, Antonio Limosani, Mike Luke, Matthias Neubert, Maurizio Pierini, Jim Smith, Iain Stewart, Stéphane T'Jampens, Nikolai Uraltsev and Matthew Wingate. I am especially grateful to Vincenzo Cirigliano and Björn Lange for their thorough and patient explanations of recent progress in the fields of chiral perturbation theory and B meson shape functions. Very special thanks go to Andreas Höcker and Heiko Lacker for their day-and-night work on the presented CKMfitter plots.

\section{References}

1. S. Eidelman et al. [Particle Data Group], Phys. Lett. B 592, 1 (2004).

2. L. Wolfenstein, Phys. Rev. Lett. 51, 1945 (1983).

3. A. J. Buras, M. E. Lautenbacher, G. Ostermaier, Phys. Rev. D 50, 3433 (1994).

4. Heavy Flavor Averaging Group, http://www.slac.stanford.edu/xorg/hfag

5. A. Höcker, H. Lacker, S. Laplace and F. Le Diberder, Eur. Phys. J. C 21, 225 
(2001) [arXiv:hep-ph/0104062]. J. Charles et al. [CKMfitter Group], Eur. Phys. J. C 41, 1 (2005) [arXiv:hep-ph/0406184]. http://www.slac.stanford.edu/xorg/ckmfitter

6. M. Ciuchini et al., JHEP 0107, 013 (2001)[arXiv:hep-ph/0012308]. M. Bona et al. [UTfit Collaboration], arXiv:hepph/0509219. http://utfit.roma1.infn.it

7. Iain Stewart, these proceeedings.

8. John Hardy, talk at KAON 2005 Int. Workshop, Jul 13-17, 2005, Evanston, USA.

9. M. Ademollo and R. Gatto, Phys. Rev. Lett. 13, 264 (1964).

10. A. Czarnecki, W. J. Marciano and A. Sirlin, Phys. Rev. D 70, 093006 (2004) [arXiv:hepph/0406324].

11. Vincenzo Cirigliano, talk at KAON 2005 Int. Workshop, Jul 13-17, 2005, Evanston, USA, and private communication.

12. J. Gasser and H. Leutwyler, Annals Phys. 158, 142 (1984).

13. V. Cirigliano, M. Knecht, H. Neufeld, H. Rupertsberger and P. Talavera, Eur. Phys. J. C 23, 121 (2002) [arXiv:hepph/0110153]. V. Cirigliano, H. Neufeld and H. Pichl, Eur. Phys. J. C 35, 53 (2004) [arXiv:hep-ph/0401173]. T. C. Andre, arXiv:hep-ph/0406006. S. Descotes-Genon and B. Moussallam, arXiv:hep-ph/0505077.

14. H. Leutwyler and M. Roos, Z. Phys. C 25, 91 (1984). P. Post and K. Schilcher, Nucl. Phys. B 599, 30 (2001) [arXiv:hepph/0007095].J. Bijnens and P. Talavera, Nucl. Phys. B 669, 341 (2003) [arXiv:hepph/0303103]. M. Jamin, J. A. Oller and A. Pich, JHEP 0402, 047 (2004) [arXiv:hep-ph/0401080]. V. Cirigliano, Int. J. Mod. Phys. A 20, 3732 (2005).

$f_{+}^{K^{0} \pi^{-}}$was computed in quenched lattice QCD in: D. Becirevic et al., Eur. Phys. J. A 24S1, 69 (2005) [arXiv:hep-lat/0411016]; V. Lubicz et al., presented at DAFNE 2004: Workshop on Physics at Meson Factories, Rome, Frascati, Italy, 7-11 Jun 2004

15. W. J. Marciano, Phys. Rev. Lett. 93, 231803 (2004) [arXiv:hep-ph/0402299].

16. C. Aubin et al. [MILC Collaboration], Nucl. Phys. Proc. Suppl. 140, 231 (2005) [arXiv:hep-lat/0409041].

17. A. Pich and J. Prades, JHEP 9806, 013 (1998) [arXiv:hep-ph/9804462]. E. Gamiz, M. Jamin, A. Pich, J. Prades and F. Schwab, JHEP 0301, 060 (2003) [arXiv:hep-ph/0212230].
18. E. Gamiz, M. Jamin, A. Pich, J. Prades and F. Schwab, Phys. Rev. Lett. 94, 011803 (2005) [arXiv:hep-ph/0408044].

19. G. Abbiendi et al. [OPAL Collaboration], Eur. Phys. J. C 35, 437 (2004) [arXiv:hepex/0406007].

20. K. R. Schubert, Int. J. Mod. Phys. A 19, 1004 (2004).

21. M. A. Shifman and M. B. Voloshin, Sov. J. Nucl. Phys. 41, 120 (1985) [Yad. Fiz. 41, 187 (1985)]. I. I. Y. Bigi, N. G. Uraltsev and A. I. Vainshtein, Phys. Lett. B 293, 430 (1992) [Erratum-ibid. B 297, 477 (1993)] [arXiv:hep-ph/9207214].

22. P. Gambino and N. Uraltsev, Eur. Phys. J. C 34, 181 (2004) [arXiv:hep-ph/0401063]. C. W. Bauer, Z. Ligeti, M. Luke, A. V. Manohar and M. Trott, Phys. Rev. D 70, 094017 (2004) [arXiv:hep-ph/0408002].

23. O. Buchmüller and H. Flächer, arXiv:hepph/0507253, and references therein.

24. C. W. Bauer and A. V. Manohar, Phys. Rev. D 70, 034024 (2004) [arXiv:hepph/0312109]. S. W. Bosch, B. O. Lange, M. Neubert and G. Paz, Phys. Rev. Lett. 93, 221801 (2004) [arXiv:hep-ph/0403223].

25. K. S. M. Lee and I. W. Stewart, Nucl. Phys. B 721, 325 (2005) [arXiv:hep-ph/0409045]. S. W. Bosch, M. Neubert and G. Paz, JHEP 0411, 073 (2004) [arXiv:hep-ph/0409115]. M. Beneke, F. Campanario, T. Mannel and B. D. Pecjak, JHEP 0506, 071 (2005) [arXiv:hep-ph/0411395].

26. B. O. Lange, M. Neubert and G. Paz, arXiv:hep-ph/0504071.

27. T. Mannel and S. Recksiegel, Phys. Rev. D 63, 094011 (2001) [arXiv:hep-ph/0009268].

28. A. K. Leibovich, I. Low and I. Z. Rothstein, Phys. Lett. B 513, 83 (2001) [arXiv:hep-ph/0105066]. A. K. Leibovich, I. Low and I. Z. Rothstein, Phys. Rev. D 61, 053006 (2000) [arXiv:hep-ph/9909404]. A. K. Leibovich, I. Low and I. Z. Rothstein, Phys. Lett. B 486, 86 (2000) [arXiv:hep$\mathrm{ph} / 0005124]$.

29. A. Bornheim et al. [CLEO Collaboration], Phys. Rev. Lett. 88, 231803 (2002) [arXiv:hep-ex/0202019].

30. H. Kakuno et al. [BELLE Collaboration], Phys. Rev. Lett. 92, 101801 (2004) [arXiv:hep-ex/0311048]. I. Bizjak et al. [Belle Collaboration], arXiv:hepex/0505088. A. Limosani et al. [Belle Collaboration], Phys. Lett. B 621, 28 (2005) 
[arXiv:hep-ex/0504046].

31. B. Aubert et al. [BaBar Collaboration], arXiv:hep-ex/0408075. B. Aubert et al. [BABAR Collaboration], Phys. Rev. Lett. 95, 111801 (2005) [arXiv:hep-ex/0506036]. B. Aubert et al. [BABAR Collaboration], arXiv:hep-ex/0509040. (The average quoted in Eq. (12) uses a slightly different, preliminary result.)

32. M. Gronau and D. London., Phys. Lett. B 253, 483 (1991). M. Gronau and D. Wyler, Phys. Lett. B 265, 172 (1991).

33. M. Gronau, Y. Grossman, N. Shuhmaher, A. Soffer and J. Zupan, Phys. Rev. D 69, 113003 (2004) [arXiv:hep-ph/0402055].

34. M. Gronau and D. London, Phys. Rev. Lett. 65, 3381 (1990).

35. E. Gamiz, S. Collins, C. T. H. Davies, J. Shigemitsu and M. Wingate, arXiv:heplat/0509188.

36. T. Becher, E. Gamiz and K. Melnikov, arXiv:hep-lat/0507033.

37. B. Aubert et al. [BABAR Collaboration], Phys. Rev. Lett. 94, 161803 (2005) [arXiv:hep-ex/0408127]. K. Abe et al. [Belle Collaboration], arXiv:hepex/0507037. R. Barate et al. [ALEPH Collaboration], Phys. Lett. B 492, 259 (2000) [arXiv:hep-ex/0009058]. K. Ackerstaff et al. [OPAL collaboration], Eur. Phys. J. C 5, 379 (1998) [arXiv:hep-ex/9801022]. T. Affolder et al. [CDF Collaboration], Phys. Rev. D 61, 072005 (2000) [arXiv:hepex/9909003].

38. A. Gray et al. [HPQCD Collaboration], arXiv:hep-lat/0507015.

39. S. Herrlich and U. Nierste, Phys. Rev. D 52, 6505 (1995) [arXiv:hep-ph/9507262].

40. G. Buchalla and A. J. Buras, Nucl. Phys. B 400, 225 (1993). G. Buchalla and A. J. Buras, M. Misiak and J. Urban, Phys. Lett. B 451, 161 (1999) [arXiv:hepph/9901278]. Nucl. Phys. B 548, 309 (1999) [arXiv:hep-ph/9901288].

41. G. Buchalla and A. J. Buras, Nucl. Phys. B 412, 106 (1994) [arXiv:hep-ph/9308272].

42. G. Buchalla and A. J. Buras, Phys. Lett. B 333, 221 (1994) [arXiv:hep-ph/9405259].

43. G. Isidori, F. Mescia and C. Smith, Nucl. Phys. B 718, 319 (2005) [arXiv:hepph/0503107]. A. F. Falk, A. Lewandowski and A. A. Petrov, Phys. Lett. B 505, 107 (2001) [arXiv:hep-ph/0012099].

44. A. J. Buras, M. Gorbahn, U. Haisch and
U. Nierste, arXiv:hep-ph/0508165.

45. M. Beneke, Phys. Lett. B 620, 143 (2005) [arXiv:hep-ph/0505075].

\section{DISCUSSION}

Luca Silvestrini (Rome and Munich): Maybe rather than saying that QCD cannot explain $\sin 2 \beta^{\text {eff }}$ in $b \rightarrow s$ penguins, one should say that a particular model of power supressed corrections due to Beneke and Co. cannot do it, but this is not a model-independent statement. If you just want to use data, and you say you do not know anything about power corrections, I do not think that you can infere anything from that plot.

Ulrich Nierste: The parametric suppression of the up-quark pollution by $\left|V_{u b} V_{u s} /\left(V_{c b} V_{c s}\right)\right| \sim 0.025$ is undisputed. Further the leading term in the $1 / m_{b}$ expansion of $\sin 2 \beta^{\text {eff }}-\sin (2 \beta)$ can be reliably computed and results in the finding of Ref. ${ }^{45}$ that $\sin 2 \beta^{\text {eff }}-\sin (2 \beta)$ is small and positive for the measured modes. It is true that the size of the modeled power corrections is currently widely debated. Yet I am not aware of any possible dynamical QCD effect in two-body B decays which is formally $\mathcal{O}\left(1 / m_{b}\right)$, large in magnitude and further comes with the large strong phase needed to flip the sign of $\sin 2 \beta^{\text {eff }}-\sin (2 \beta)$. 